\title{
Étude comparative de la dégradation du bromoxynil et du bromoxynil heptanoate par photolyse UV et par oxydation chimique $\left(\mathrm{H}^{2} \mathrm{O}^{2} / \mathrm{UV} ; \mathrm{O}^{3} ; \mathrm{Cl}^{2}\right)$ \\ Comparative Study of the Degradation of Bromoxynil and Bromoxynil heptanoate by UV photolysis and by Chemical Oxidation (H2O2/UV; O3; Cl2)
}

\author{
S. Guitonneau, S. Momege, A. Schafmeier, P. O. Viac et P. Meallier
}

Volume 8, numéro 2, 1995

URI : https://id.erudit.org/iderudit/705219ar

DOI : https://doi.org/10.7202/705219ar

Aller au sommaire du numéro

\section{Éditeur(s)}

Université du Québec - INRS-Eau, Terre et Environnement (INRS-ETE)

\section{ISSN}

0992-7158 (imprimé)

1718-8598 (numérique)

Découvrir la revue

Citer cet article

Guitonneau, S., Momege, S., Schafmeier, A., Viac, P. O. \& Meallier, P. (1995).

Étude comparative de la dégradation du bromoxynil et du bromoxynil

heptanoate par photolyse UV et par oxydation chimique $\left(\mathrm{H}^{2} \mathrm{O}^{2} / \mathrm{UV} ; \mathrm{O}^{3} ; \mathrm{Cl}^{2}\right)$.

Revue des sciences de l'eau / Journal of Water Science, 8(2), 201-216.

https://doi.org/10.7202/705219ar

\section{Résumé de l'article}

Les dégradations du bromoxynil heptanoate et du bromoxynil ont été étudiées pour différents traitements chimiques $(03, \mathrm{Cl} 2)$ et photochimiques $(\mathrm{H} 202 / \mathrm{W}$; W254) en milieu aqueux dilué. Les expériences ont été réalisées à $\mathrm{pH} 7$ pour le bromoxynil $(\mathrm{CO}=5$ 10-5 mol 1-1) et à $\mathrm{pH} 4$ pour le bromoxynil heptanoate ( $\mathrm{CO}=10-6 \mathrm{~mol} 1-1)$ afin de limiter la réaction d'hydrolyse.

Les résultats obtenus montrent une bonne dégradation de ces deux pesticides aussi bien par irradiation $\mathrm{W}$ que par oxydation chimique au chlore ou à l'ozone. L'ajout de peroxyde d'hydrogène dans le milieu n'augmente pas de façon significative l'efficacité du traitement par rapport à l'irradiation seule. Parmi tous les traitements étudiés, l'ozonation est le procédé le plus efficace. L'analyse par couplage GC-MS des produits de réaction au cours de l'irradiation UV a permis d'identifier quatre photoproduits correspondant à la substitution des atomes de brome soit par un atome d'hydrogène, soit par un groupement OH. Les produits identifés sont A: -3,4 dihydroxy -5 bromobenzonitrile; B: -3,4,5 trihydroxybenzonitrile; C: -3,4 dihydroxybenzonitrile; D: -4 hydroxybenzonitrile. Un schéma réactionnel de photodégradation du bromoxynil heptanoate a été proposé dans cette étude.
Tous droits réservés ( Revue des sciences de l'eau, 1995
Ce document est protégé par la loi sur le droit d'auteur. L’utilisation des services d'Érudit (y compris la reproduction) est assujettie à sa politique d'utilisation que vous pouvez consulter en ligne.

https://apropos.erudit.org/fr/usagers/politique-dutilisation/ 


\title{
Etude comparative de la dégradation du bromoxynil et du bromoxynil heptanoate par photolyse UV et par oxydation chimique $\left(\mathrm{H}_{2} \mathrm{O}_{2} / \mathrm{UV} ; \mathrm{O}_{3} ; \mathrm{Cl}_{2}\right)$
}

\author{
Comparative study of the degradation of bromoxynil \\ and bromoxynil heptanoate by UV photolysis \\ and by chemical oxidation $-\left(\mathrm{H}_{2} \mathrm{O}_{2} / \mathrm{UV} ; \mathrm{O}_{3} ; \mathrm{Cl}_{2}\right)$
}

\section{S. GUITTONNEAU', S. MOMEGE, A. SCHAFMEIER, P.O. VIAC ET P. MEALLIER}

Reçu le 25 octobre 1994, accepté le 5 décembre 1994*.

\section{SUMMARY}

In the aquatic environment hydrolysis and phototransformation processes are the main abiotic degradation routes of chemicals. The aim of this study is to investigate the degradation pathway of 2 pesticides (bromoxynil heptanoate and its hy drolysis product, bromoxynil) by UV photolysis and by chemical oxidation. The chemical oxidation processes studied are ozonation, chlorination and radical oxidation with $\mathrm{OH}$ radicals. These radicals are produced by photolysis of hydrogen peroxide.

Bromoxynil heptanoate ( -4 cyano $-2,6$ dibromophenyl heptanoate) is an ester which is quite unstable in aqueous medium. Preliminary experiments, carried out in diluted solution $\left(C_{0}=10^{-6} \mathrm{~mol} 1-1\right)$ and in phosphate buffer $\left(\mu=10^{-2} \mathrm{~mol} \mathrm{l}-1\right)$, showed that 20 to $25 \%$ of bromoxynil heptanoate is hydrolyzed after 24 hours at pH 7 . The hydrolysis decreases strongty with pH; it is only $3 \%$ at pH 4 after one day.

In order to limit the hydrolysis reaction, the following experiments were carried out at pH 7 for bromoxynil $\left(\mathrm{Co}=510^{-5} \mathrm{~mol} \mathrm{1-1}\right)$ and at $\mathrm{pH} 4$ for bromoxynil heptanoate $\left(\mathrm{Co}=10^{-6} \mathrm{~mol} \mathrm{l}^{-1}\right)$. The $\mathrm{pH}$ was adjusted with a phosphate buffer.

\begin{abstract}
Absorption spectra of bromoxynil heptanoate and bromoxynil do not show any absorption band up to $320 \mathrm{~nm}$ (bromoxynil heptanoate 1 max $=218,290 \mathrm{~nm}$; bromoxynil I $\max =222,280 \mathrm{~nm}$; fig. 1). Therefore, the removal of these two pesticides by direct photolysis with the sun light will be very small. The measurement of the quantum yield (number of molecules undergoing photodegradation trans formation per number of photons absorbed by these molecules) in the UV region in both monochromatic and polychromatic lights (HPK, Philips; $I_{0}=1.4810-8$ Einstein sec-1), indicates that these 2 compounds are easily eliminated by a UV irradiation treatment $\left(10^{-2}<\mathrm{F}<10^{-1}\right.$ at $\mathrm{I}=254 \mathrm{~nm}$ and $\left.290 \mathrm{~nm}\right)$.
\end{abstract}

1. Laboratoire de Photochimie industrielle, Université Claude Bernard, Lyon I, 43 boulevard du 11 novembre 1918 , 69622 Villeurbanne Cedex.

* Les commentaires seront reçus jusqu'au 24 janvier 1996. 
The irradiation of these two pesticides at $254 \mathrm{~nm}$ (low pressure vapor mercury $\operatorname{lamp}, I_{0}=5.810^{-8}$ Einstein sec-1) in the presence of hydrogen peroxide does not seem to significantly improve the efficiency of the treatment by UV irradiation alone. For example, the half life time of bromoxynil is 11 minutes by UV compared to 8 minutes when hydrogen peroxide is present. This small difference of reactivity of $\mathrm{H}_{2} \mathrm{O}_{2} / \mathrm{UV}$ system is due to the small quantity of $\mathrm{H}_{2} \mathrm{O}_{2}$ photolyzed during this irradiation time; at $\mathrm{t}=8 \mathrm{~min}$ only $1 \%$ of $\mathrm{H}_{2} \mathrm{O}_{2}$ is decomposed.

The oxidation of bromoxynil heptanoate and bromoxynil by ozonation was performed in a semi-batch reactor $\left(Q=5 \mathrm{mg} \mathrm{O}_{3} \mathrm{~min}^{-1}\right)$. Results show that these two compounds are very reactive with ozone. $50 \%$ of elimination was obtained after 5 and 2 minutes of ozonation for bromoxynil heptanoate and bromoxynil respectively. In both cases the transfer of ozone was the limiting factor.

Finally chlorination of bromoxynil at a rate of $35.5 \mathrm{mg} \mathrm{Cl}_{2} \mathrm{I}^{-1}$ indicates that this pesticide is also oxidized by chlorine but the degradation rate is quite slow; a total degradation is observed after $90 \mathrm{~min}$ of reaction.

Comparison of the different treatments studied shows that ozonation is certainly the most effective process. Only a few minutes with reasonable ozone doses are necessary to eliminate these pesticides from aqueous medium. The other treatment $\left(\mathrm{UV}, \mathrm{H}_{2} \mathrm{O}_{2} / \mathrm{UV}, \mathrm{Cl}_{2}\right.$ ) can also be a good alternative for the removal of these compounds from water.

For the different oxidation treatments, the HPLC analysis of the bromoxynil reaction mixture shows the formation of by-products. Only $\mathrm{UV}$ and $\mathrm{H}_{2} \mathrm{O}_{2} / \mathrm{UV}$ byproducts were identified in this work; but comparison of the retention times from the HPLC analysis indicates that certain by-products from ozonation are similar to the ones observed by UV and $\mathrm{H}_{2} \mathrm{O}_{2} / \mathrm{UV}$ oxidation. On the other hand, the products formed during chlorination, probably chlorinated products, have different retention times.

The reaction intermediates were identified by GC-MS analysis after $10 \mathrm{~min}$ of irradiation (with and without $\mathrm{H}_{2} \mathrm{O}_{2}$ ). This time corresponds to the maximum con centration of one of the photoproduct. Two kinds of compounds were identified from the GC-MS analysis: hydroxylated compounds $(-3,4$ dihydroxy -5 bromobenzonitrile; $-3,4,5$ trihydroxybenzonitrile) and hydrogenated compounds $(-3,4$ dihydroxybenzonitrile; -4 hydroxybenzonitrile). In both cases the bromine atoms were substituted either by an $\mathrm{H}$ atom or by an $\mathrm{OH}$ group. A reaction mechanism of photodegradation of bromoxynil heptanoate is proposed in this study (Fig. 6).

Key-words : bromoxynil ( $-3,5$ dibromobenzonitrile), bromoxynil heptanoate ( -4 cyano $-2,6$ dibromophenyl heptanoate), ozonation, chlorination, quantum yield, $\mathrm{H}_{2} \mathrm{O} / \mathrm{UV}$ oxidation, aqueous medium, by-products.

Les dégradations du bromoxynil heptanoate et du bromoxynil ont été étudiées pour différents traitements chimiques $\left(\mathrm{O}_{3}, \mathrm{Cl}_{2}\right)$ et photochimiques $\left(\mathrm{H}_{2} \mathrm{O}_{2} / \mathrm{UV}\right.$; $\mathrm{UV}_{254}$ ) en milieu aqueux dilué. Les expériences ont été réalisées à $\mathrm{pH} 7$ pour le bromoxynil $\left(C_{0}=510^{-5} \mathrm{~mol} \mathrm{l}^{-1}\right)$ et à $\mathrm{pH} 4$ pour le bromoxynil heptanoate $\left(C_{0}=10^{-6} \mathrm{~mol} \mathrm{l}^{-1}\right)$ afin de limiter la réaction d'hydrolyse.

Les résultats obtenus montrent une bonne dégradation de ces deux pesticides aussi bien par irradiation UV que par oxydation chimique au chlore ou à l'ozone. L'ajout de peroxyde d'hydrogène dans le milieu n'augmente pas de façon significative l'efficacité du traitement par rapport à l'irradiation seule. Parmi tous les traitements étudiés, l'ozonation est le procédé le plus efficace. 
L'analyse par couplage GC-MS des produits de réaction au cours de l'ìrradiation UV a permis d'identifier quatre photoproduits correspondant à la substitution des atomes de brome soit par un atome d'hydrogène, soit par un groupement $\mathrm{OH}$. Les produits identifés sont $A:-3,4$ dihydroxy $\mathbf{- 5}$ bromobenzonitrile ; $B: \mathbf{- 3 , 4 , 5}$ trihydroxybenzonitrile; $\mathrm{C}: \mathbf{- 3 , 4}$ dihydroxybenzonitrile ; $\mathrm{D}:-4$ hydroxybenzonitrile. Un schéma réactionnel de photodégradation du bromoxynil heptanoate a été proposé dans cette étude.

Mots clés : bromoxynil (-3,5 dibromobenzonitrile), bromoxynil heptanoate ( -4 cyano $-2,6$ dibromophényl heptanoate), ozonation, chloration, rendement quantique, sousproduits de réaction.

\section{INTRODUCTION}

Préalablement à leur mise sur le marché, les produits phytosanitaires doivent avoir fait l'objet d'une homologation accordée notamment sur la base d'études établissant l'innocuité du produit pour l'homme et l'environnement. Le bromoxynil et le bromoxynil heptanoate sont deux herbicides commercialisés par la société Rhône-Poulenc. Ils sont utilisés en traitement de post levée dans la culture des céréales et agissent par contact sélectif en bloquant la photosynthèse.

La solubilité dans l'eau de ces deux composés est faible, elle est égale à $130 \mathrm{mg} \mathrm{l}^{-1}$ pour le bromoxynil et est inférieure à $1 \mathrm{mg} \mathrm{l}^{-1}$ pour le bromoxynil heptanoate. Les concentrations susceptibles d'être retrouvées dans les eaux superficielles seront donc peu élevées, mais elles seront néanmoins suffisantes pour que l'on s'intéresse à leur devenir dans l'eau, compte tenu de la concentration maximale admissible dans l'eau potable fixée par la directive européenne à $0,1 \mu \mathrm{g} \mathrm{l}^{-1}$ (80/778/CEE).

Dans le milieu aquatique, la biodégradation, l'hydrolyse ainsi que les processus de phototransformation directe ou indirecte seront les principales voies de dégradation de ces produits chimiques. L'évolution du bromoxynil et de ses formes estérifiées (octanoate et butyrate) dans des conditions similaires à celles du milieu naturel ont fait l'objet de quelques travaux en littérature. Ces études montrent que les composés de la famille du bromoxynil se décomposent par réaction d'hydrolyse (BROWN et al., 1984) et par photodégradation directe ou indirecte à $313 \mathrm{~nm}$ (KOCHANY et al., 1990 (a)). Mais étant donné la faible absorption de ces produits dans le visible le risque de les retrouver dans une eau de surface est réel.

Dans ce travail nous nous sommes intéressés à l'élimination du bromoxynil heptanoate et de son produit d'hydrolyse, le bromoxynil, par des traitements chimiques et photochimiques dans des conditions proches de celles d'une station d'eau potable. Les procédés chimiques étudiés sont des procédés d'oxydation à l'ozone, au chlore et radicalaire, faisant intervenir les radicaux $\mathrm{OH}^{\circ}$; ces radicaux ont été formés à partir du couplage $\mathrm{H}_{2} \mathrm{O}_{2} / U V$. 


\section{MATÉRIEL ET MÉTHODES}

\section{Préparation des solutions}

Les expériences ont été réalisées avec des solutions aqueuses préparées dans de l'eau ultrapure (Milli $Q$ ), tamponnées à partir de sels de phosphate $\left(\mu=10^{-2} \mathrm{~mol} \mathrm{l}^{-1}\right)$. Etant donné la faible solubilité du bromoxynil heptanoate dans l'eau, les solutions ont été préparées par saturation de l'eau tamponnée par le pesticide sous agitation pendant 12 heures, suivie d'une filtration. Les concentrations des solutions ainsi préparées varient entre $4 \quad 10^{-7}$ et $1,510^{-6} \mathrm{~mol} \mathrm{I}^{-1}$. La concentration exacte de la solution a été déterminée par analyse HPLC avec un étalonnage préparé dans te méthanol.

Les solutions de bromoxynil en milieu aqueux, ont été préparées directement à la concentration voulue pour des valeurs inférieures à $510^{-4} \mathrm{~mol} \mathrm{l}^{-1}$.

\section{Réacteurs}

\section{Irradiations}

Les irradiations des solutions de pesticides, avec ou sans $\mathrm{H}_{2} \mathrm{O}_{2}$, ont été réalisées dans un photoréacteur annulaire (Applied Photophysics, modèle $A P Q 40)$. Ce réacteur est équipé d'un carrousel dans lequel sont répartis 9 tubes en quartz contenant la solution à irradier $(\varnothing=1 \mathrm{~cm} ; \mathrm{V}=15 \mathrm{ml})$. Les tubes tournent autour d'une lampe basse pression à vapeur de mercure (Applied Photophysics, modèle 3016, $16 \mathrm{~W}$ ) refroidie par une circulation d'eau dans une double enveloppe. Pour chaque temps de réaction, un tube a été prélevé pour les analyses. Le flux lumineux total reçu par chaque tube est de $5,710^{-8}$ Einstein $\mathrm{sec}^{-1}$ et de $3,910^{-8}$ Einstein $\mathrm{sec}^{-1}$ à $254 \mathrm{~nm}$. Ces énergies ont été déterminées par actinométrie chimique à l'oxalate d'uranyle pour l'émission totale (BRAUN et al., 1986) et à partir du peroxyde d'hydrogène pour l'énergie à $254 \mathrm{~nm}$ (NICOLE et al., 1990).

Les rendements quantiques de photodégradation ont été déterminés sur un banc optique équipé d'une lampe haute pression à vapeur de mercure (Philips, HPK 125), d'un monochromateur et de deux cellules en quartz refroidies par une circulation d'eau. Les flux lumineux ont été mesurés en utilisant roxalate d'uranyle comme actinomètre.

\section{Ozonation}

Les expériences d'ozonation ont été réalisées dans un réacteur semibatch, de capacité utile de 1,8 litre. L'ozone est préparé par passage d'oxygène dans un diélectrique à un débit de $10 \mathrm{ml} \mathrm{sec}^{-1}$ et est introduit en continu dans le réacteur à partir d'un diffuseur. Une agitation permet d'assurer l'homogénéité de la solution.

Au cours des diverses expériences, les flux d'ozone gazeux ont été contrôlés à partir de barbotteurs contenant une solution de iodure de potassium à $20 \mathrm{~g} \mathrm{l}^{-1}$, placés à l'entrée et à la sortie du réacteur. Les prélèvements de la solution ozonée ont été réalisés à l'aide d'une vanne située à la base 
du réacteur d'ozonation. Les concentrations d'ozone mesurées sont de $8,33 \mathrm{mg} \mathrm{t}^{-1}$ dans les gaz et de $2,5 \mathrm{mg} \mathrm{l}^{-1}$ en ozone résiduel dans la solution $\left(\mathrm{pH}=7 ; \mu=10^{-2} \mathrm{~mol}^{-1}\right)$.

\section{Chloration}

Les réactions de chloration ont été effectuées sous agitation sur un volume total de $250 \mathrm{ml}$ par injection d'une solution concentrée d'eau de javel à la solution de pesticide.

Pour chaque temps de cohtact, un volume de solution a été prélevé pour les analyses et dans le cas du dosage du produit organique, la réaction a été stoppée avec quelques gouttes $(0,2 \mathrm{ml})$ de thiosulfate de sodium à $0,1 \mathrm{~mol}^{-1}$.

\section{Dosages}

\section{Dosage des produits organiques}

Les concentrations en bromoxynil et bromoxynil heptanoate ont été déterminées par HPLC (pompe Waters, modèle 510) avec une détection UV (Spectra Physics, modèle UV 2000). Les conditions d'analyses ont été reportées dans le tableau 1.

Tableau 1 Conditions d'analyse chromatographique du bromoxynil et du bromoxynil heptanoate.

Table 1 HPLC operating conditions for bromoxynil and bromoxynil heptanoate analysis.

\begin{tabular}{|c|c|c|}
\hline & Bromoxynil & Bromoxynil heptanoate \\
\hline Colonne & $C_{18}$ ODS 2 & $C_{18}$ RSIL \\
\hline Phase mobile & Méthanol/eau/ $\mathrm{CH}_{3} \mathrm{COOH}: 55 / 44 / 1$ & Méthanoveau : $90 / 10$ \\
\hline Débit & $1 \mathrm{~m}^{\text {min }}{ }^{-1}$ & $1 \mathrm{mlmin} \mathrm{m}^{-1}$ \\
\hline$\lambda(\mathrm{nm})$ & 254 & 212 \\
\hline $\operatorname{Tr}(\min )$ & 11,5 & 7,5 \\
\hline $\begin{array}{l}\text { Limite de détection } \\
\left(\text { molt }^{-1}\right)\end{array}$ & $510^{-7}$ & $0,510^{-7}$ \\
\hline
\end{tabular}

\section{Dosage des oxydants}

Les concentrations en peroxyde d'hydrogène et en ozone ont été mesurées au cours du temps de la réaction étudiée par des méthodes spectroscopiques. Les concentrations de $\mathrm{H}_{2} \mathrm{O}_{2}$ ont été déterminées par la méthode colorimétrique au titane à $410 \mathrm{~nm}$ (EISENBERG, 1943) et l'ozone en solution par la méthode au carmin indigo à $\lambda=600 \mathrm{~nm}$ (BADER et HOIGNE, 1981).

Les concentrations en chlore en solution et en ozone dans les gaz ont été déterminées par dosage iodométrique (STANDARDISATION COMMITTEE, 1987). 


\section{Protocole d'extraction et d'identification des sous-produits de réaction}

L'analyse des produits intermédiaires d'oxydation du bromoxynil $\left(\mathrm{C}_{0}=\right.$ $\left.210^{-4} \mathrm{~mol}^{-1}\right)$ a nécessité une extraction et une réaction de dérivation de ces composés avant l'identification par couplage GC - MS.

Le protocole suivi a été le suivant : un échantillon de $100 \mathrm{ml}$ de produits à analyser a été évaporé à sec puis repris avec $10 \mathrm{ml}$ d'acétonitrile. Cette solution a été ensuite concentrée 10 fois avant d'effectuer la réaction de silylation au BSTFA (bis-triméthylsilyl trifluoroacétamide). L'échantillon ainsi préparé a ensuite été injecté en chromatographie gazeuse sur une colonne capillaire apolaire (DB5) avec une rampe de température de $70^{\circ} \mathrm{C}$ pendant $10 \mathrm{~min}$; $15^{\circ} \mathrm{C} \mathrm{min-1}$ jusqu'à $150^{\circ} \mathrm{C}$ puis $10^{\circ} \mathrm{C} \mathrm{min}-1$ jusqu'à $250^{\circ} \mathrm{C}$ et enfin un palier de $10 \mathrm{~min}$ à cette température.

Lorsque $\mathrm{H}_{2} \mathrm{O}_{2}$ était présent dans le milieu, celui-ci a été détruit préalablement par quelques gouttes de thiosulfate avant d'effectuer l'évaporation à sec de la solution.

\section{RÉSULTATS EXPÉRIMENTAUX}

Le bromoxynil heptanoate est un ester relativement instable en milieu aqueux. Une étude préliminaire réalisée sur des solutions à $10^{-6} \mathrm{~mol} \mathrm{I}^{-1}$ en pesticide et dans un tampon phosphate $\left(\mu=10^{-2} \mathrm{~mol}^{-1}\right)$ a montré que de 20 à $25 \%$ du produit est hydrolysé à pH 7 au bout de 24 heures. Cette hydrolyse diminue fortement avec le $\mathrm{pH}$, elle est inférieure à $3 \%$ à $\mathrm{pH} 4$ pour ce même temps. Pour éviter la disparition du bromoxynil heptanoate, les études ont été réalisées sur des solutions aqueuses diluées à $\mathrm{pH} 4$ pour le bromoxynil heptanoate $\left(\mathrm{C}_{\mathrm{o}}=10^{-6} \mathrm{~mol} \mathrm{l}^{-1}\right)$ et à $\mathrm{pH} 7$ pour le bromoxynil $\left(\mathrm{C}_{\mathrm{o}}=\right.$ $\left.510^{-5} \mathrm{~mol} \mathrm{I}^{-1}\right)$.

\section{Détermination du rendement quantique de photodégradation UV du bromoxynil et du bromoxynil heptanoate}

Le tracé des spectres d'absorption du bromoxynil et du bromoxynil heptanoate en solution méthanolique montre deux bandes d'absorption (fig. 1), une première bande autour de $290 \mathrm{~nm}$ (bromoxynil : $\varepsilon_{280}=20000 \mathrm{I} \mathrm{mol}^{-1} \mathrm{~cm}^{-1}$; bromoxynil heptanoate : $\varepsilon_{290}=500 \mathrm{I} \mathrm{mol}^{-1} \mathrm{~cm}^{-1}$ ) et une seconde autour de $220 \mathrm{~nm}$ (bromoxynil : $\varepsilon_{222}=220001 \mathrm{~mol}^{-1} \mathrm{~cm}^{-1}$; bromoxynil heptanoate : $\left.\varepsilon_{218}=21500 \mathrm{I} \mathrm{mol}^{-1} \mathrm{~cm}^{-1}\right)$.

La mesure des rendements quantiques de photodégradation de ces deux pesticides (tabl. 2) a été réalisée dans l'eau et le méthanol sur la première bande d'absorption de la molécule (niveau excité $S_{1}$ ) ainsi qu'à $254 \mathrm{~nm}$, longueur d'onde correspondant à la longueur d'onde d'émission de la lampe 
utilisée pour le procédé $\mathrm{H}_{2} \mathrm{O}_{2} / \mathrm{UV}$. Pour le bromoxynil heptanoate, une efficacité quantique a également été mesurée en utilisant tout le spectre de la lampe à vapeur de mercure (lampe Philipps, HPK).

Les rendements quantiques reportés dans le tableau 2 traduisent l'instabilité de ces deux molécules sous une irradiation ultraviolette. L'absence de bande d'absorption au delà de $300 \mathrm{~nm}$ indique que le bromoxynil heptanoate ne pourra pas être dégradé par photolyse directe dans l'environnement. Par contre, son hydrolyse en bromoxynil permet d'envisager son élimination par voie photochimique étant donné que la bande d'absorption de ce dernier s'étend jusqu'à $320 \mathrm{~nm}$.

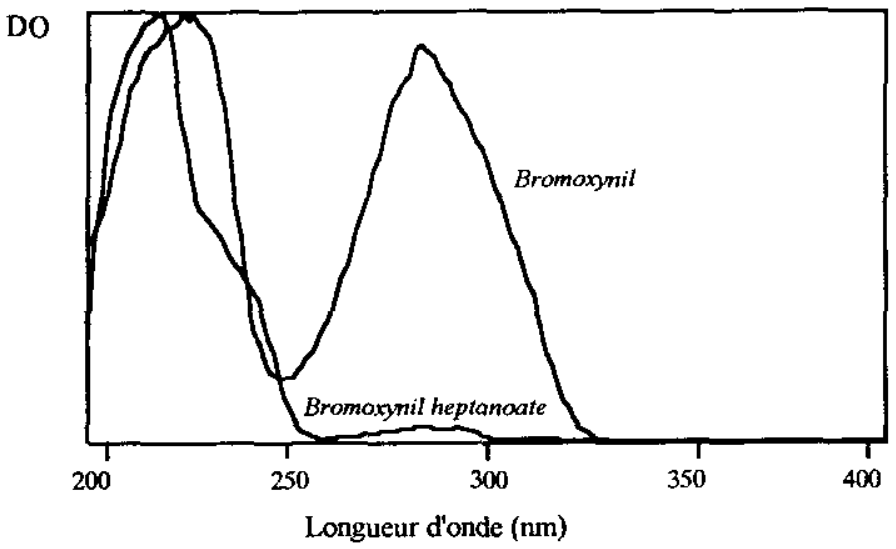

Figure 1 Spectres d'absorption du bromoxynil et du bromoxynil heptanoate Absorption spectra of bromoxynil and bromoxynil heptanoate

Tableau 2 Rendement quantique de photodégradation du bromoxynil et du bromoxynil heptanoate.

Table 2 Quantum yields for bromoxynil and bromoxynil heptanoate.

\begin{tabular}{|llrrr|}
\hline & \multicolumn{2}{c}{ Bromoxynil heptanoate } & \multicolumn{2}{c|}{ Bromoxynil } \\
\cline { 2 - 5 } \multicolumn{1}{r}{$\lambda$} & Eaul & Méthanol & Eau & Méthanol \\
\hline $254 \mathrm{~nm}$ & $10^{-2}$ & $0,810^{-2}$ & $210^{-2}$ & - \\
$290 \mathrm{~mm}$ & - & $510^{-2}$ & $210^{-2}$ & $610^{-2}$ \\
Polychromatique & - & $10^{-1}$ & - & - \\
\hline
\end{tabular}




\section{Oxydation radicalaire du bromoxynil et du bromoxynil heptanoate $\mathrm{par} \mathrm{H}_{2} \mathrm{O}_{2} / \mathrm{UV}$}

L'oxydation du bromoxynil et du bromoxynil heptanoate par le systeme $\mathrm{H}_{2} \mathrm{O}_{2}$ /UV a été réalisée en utilisant une lampe à vapeur de mercure basse pression et une solution de $\mathrm{H}_{2} \mathrm{O}_{2}$ à $410^{-4} \mathrm{~mol}^{-1}$. Etant donné la faible stabilité photochimique de ces produits organiques, un essai sans peroxyde d'hydrogène (UV seul) a été effectué afin d'évaluer l'efficacité du traitement radicalaire.

Comme le montre les figures 2 et 3 , la cinétique de dégradation de ces deux pesticides, n'est päs significativement améliorée par la présence de peroxyde d'hydrogène dans le milieu. La faible quantité de $\mathrm{H}_{2} \mathrm{O}_{2}$ photolysé pendant le temps de réaction (15\% au bout d'une heure) permet d'expliquer ces résultats.

L'analyse HPLC a permis de détecter six produits de réaction. L'évolution des concentrations de ces produits au cours du temps (fig. 4) est similaire pour le système $\mathrm{H}_{2} \mathrm{O}_{2}$ UV et les UV seuls.

\section{Étude de l'oxydation du bromoxynil et du bromoxynil heptanoate par ozonation}

Les résultats obtenus au cours de l'ozonation en réacteur à bulle $(Q=5 \mathrm{mg}$ $\mathrm{O}_{3} \mathrm{~min}^{-1}$ ) de ces deux composés ont été reportés sur la figure 5 . Ces résultats montrent que le bromoxynil heptanoate est éliminé plus lentement par l'ozone contrairement à son homologue hydroxylé. L'élimination totale du bromoxynil

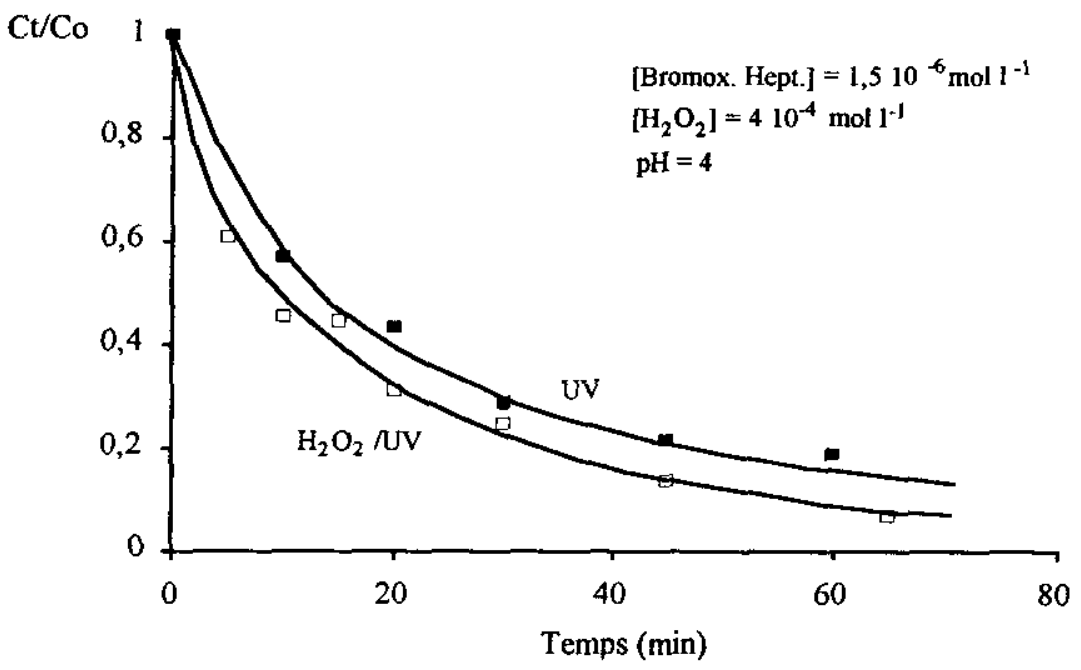

Figure 2 Photolyse UV et oxydation par $\mathrm{H}_{2} \mathrm{O}_{2} / \mathrm{UV}$ du bromoxynil heptanoate.

Removal of bromoxynil heptanoate by $\mathrm{H}_{2} \mathrm{O}_{2} \mathrm{UV}$ and by UV photolysis. 


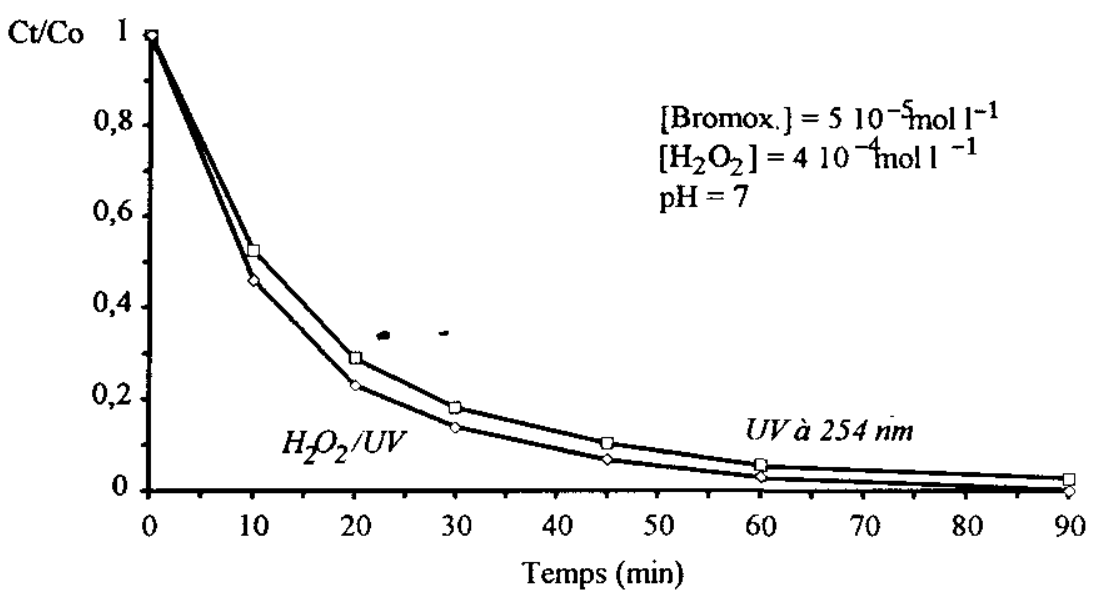

Figure 3 Photolyse UV et oxydation par $\mathrm{H}_{2} \mathrm{O}_{2} / U V$ du bromoxynil.

Removal of bromoxynil by $\mathrm{H}_{2} \mathrm{O}_{2} \mathrm{UV}$ and by $\mathrm{UV}$ photolysis.

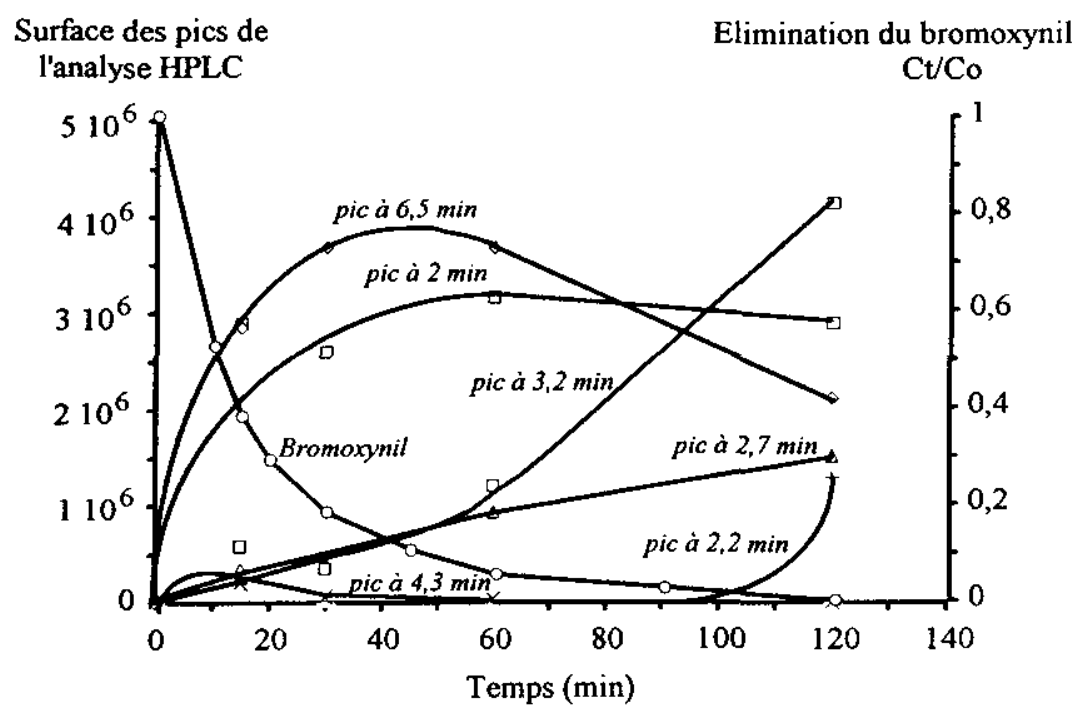

Figure 4 Evolution de la concentration des sous-produits de réaction par irradiation UV du bromoxynil.

Bromoxynil by-product formation as a function of UV irradiation time. 


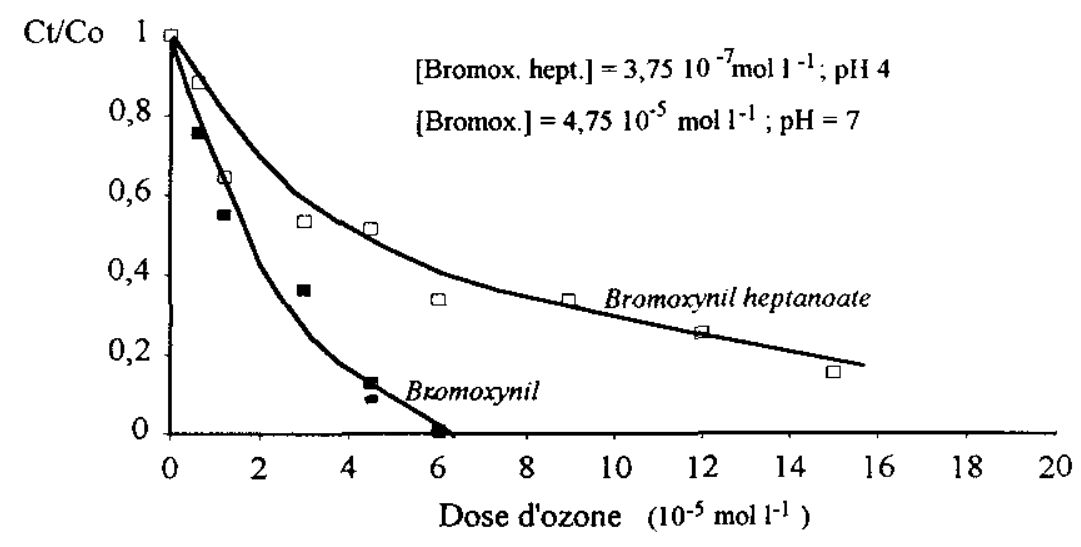

Figure 5 Ozonation du bromoxynil heptanoate et du bromoxynil.

Ozonation of bromoxynil heptanoate and bromoxynil.

est obtenue au bout de 7 minutes d'ozonation, alors que l'on a seulement $70 \%$ d'élimination du bromoxynil heptanoate pour le même temps. II est à noter que l'ozonation de ces deux produits a été réalisée à des concentrations initiales différentes ainsi qu'à des pH différents pour éviter l'hydrolyse du bromoxynil heptanoate. Pour l'oxydation du bromoxynil, il faut prendre en compte, en plus des réactions directes de l'ozone, la participation des radicaux $\mathrm{OH}^{\bullet}$ issus de la décomposition de l'ozone à $\mathrm{pH} 7$.

L'analyse HPLC du bromoxynil indique également la formation de produits de réaction qui sont eux mêmes rapidement oxydés. Le suivi du spectre d'absorption de la solution de bromoxynil au cours de l'ozonation (fig. 6) indique une oxydation poussée de ce composé, les produits de fin de réaction sont probablement des produits d'ouverture du cycle aromatique.

\section{Étude de la chloration du bromoxynil}

Seule l'oxydation du bromoxynil par le chlore a été étudiée au cours de ce travail. La réaction a été réalisée pour une dose de chlore de $35,5 \mathrm{mg} \mathrm{I}^{-1}$ et à un $\mathrm{pH} 7$.

Les résultats obtenus (fig. 7) montrent que le bromoxynil est oxydé par le chlore, une élimination presque totale du produit initial est observée au bout de 90 minutes. Nous pouvons cependant remarquer que cette oxydation est plus lente que l'ozonation.

Un seul produit de réaction a pu être détecté par HPLC (pic à $10 \mathrm{~min}$ ). $\mathrm{Ce}$ produit est probablement chloré car il a un temps de rétention différent de ceux observés pour les autres traitements d'oxydation. Le suivi de sa concentration au cours du temps montre que ce composé passe par un maximum puis disparaît du milieu. 


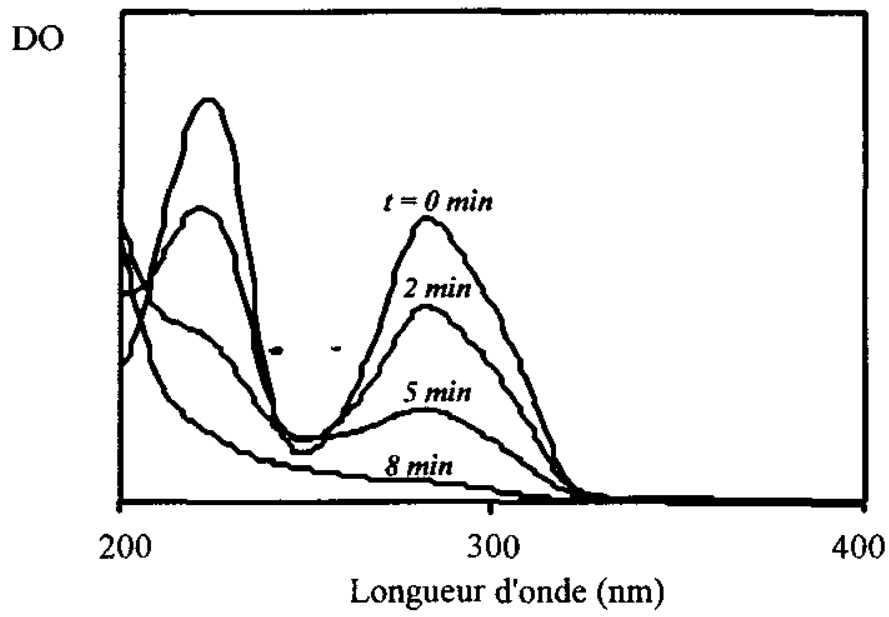

Figure 6 Spectres d'absorption des solutions ozonées de bromoxynil. Absorption spectra of ozonated bromoxynil solutions.

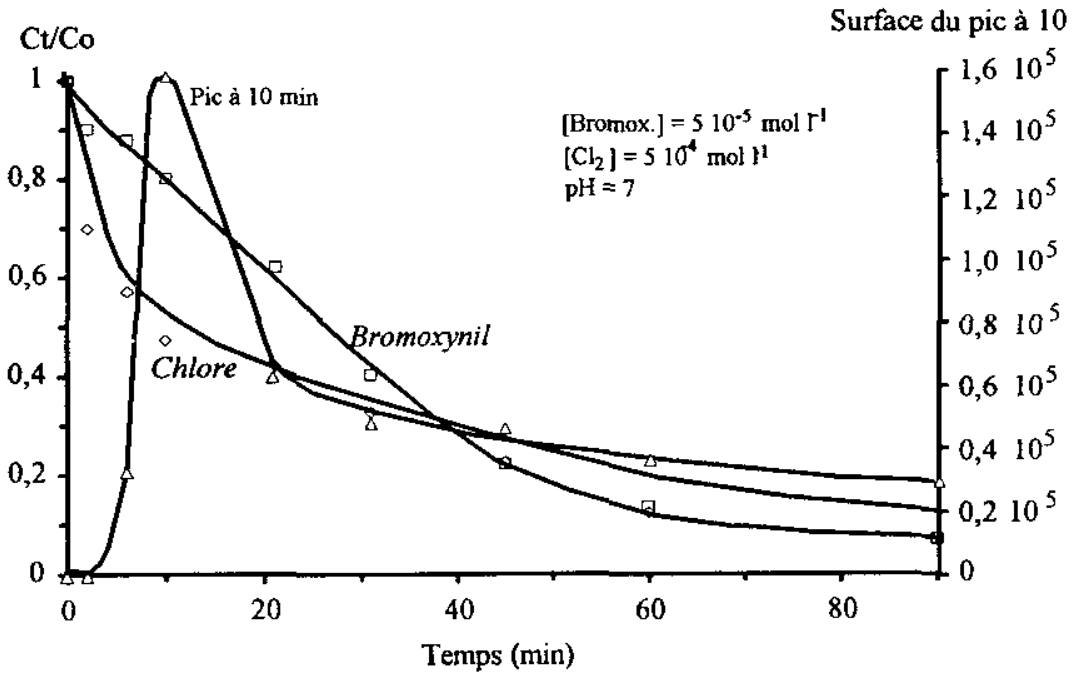

Figure 7 Chloration du bromoxynil.

Chlorination of bromoxynil.

\section{Étude des produits de réaction}

La formation des produits de réaction a été étudiée sur des solutions de bromoxynil à $210^{-4}$ mol l-1. L'irradiation de ces solutions avec ou sans $\mathrm{H}_{2} \mathrm{O}_{2}$ conduit après quelques minutes d'oxydation à une coloration brune des solutions témoignant de la présence de produits aromatiques hydroxylés. Parmi ces produits, six ont pu être détectés au cours de l'analyse HPLC, la 
figure 4 montre l'évolution de leur concentration au cours du temps. L'analyse par couplage GC-MS a permis d'identifier quatre de ces produits après dix minutes d'irradiation, ce temps correspond au maximum de concentration de l'un des photoproduits. L'analyse des intermédiaires pour un temps plus long n'a pas été réalisée car les concentrations des produits de réaction reportés dans la figure 4 , ne montrent pas d'évolution importante dans la composition de la solution.

\section{formules chimiques $\quad-$}

Produit A : $-3,4$ dihydroxy -5 benzonitrile<smiles>N#Cc1cc(O)c(O)c(Br)c1</smiles>

Produit B : $-3,4,5$ trihydroxy benzonitrile<smiles>N#Cc1cc(O)c(O)c(O)c1</smiles>

Produit $\mathrm{C}:-3,4$ dihydroxy benzonitrile<smiles>N#Cc1ccc(O)c(O)c1</smiles>

Produit D : -4 hydroxy benzonitrile

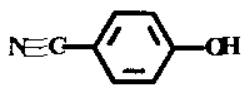

On retrouve les mêmes composés pour le système $\mathrm{H}_{2} \mathrm{O}_{2} / \mathrm{UV}$ et l'irradiation UV. Aucun produit d'ouverture du cycle n'a été détecté pour ce temps d'irradiation $(\mathrm{t}=10 \mathrm{~min})$.

\section{DISCUSSION}

Le bromoxynil heptanoate est un ester relativement instable dans l'eau qui s'hydrolyse en bromoxynil à pH 7 (20 à $25 \%$ d'élimination au bout de 24 heures):

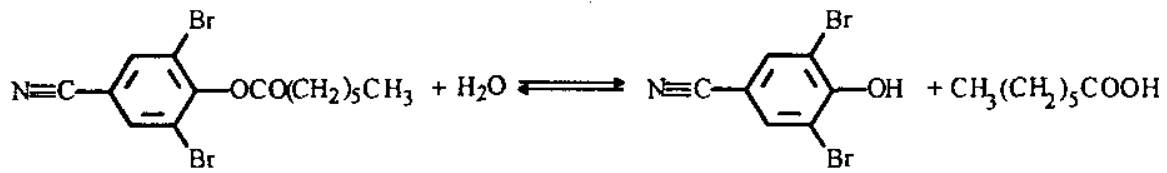


L'hydrolyse de ce composé est fortement ralentie à $\mathrm{pH}$ acide et devient pratiquement nulle à $\mathrm{pH} 4$ (moins de $3 \%$ d'élimination pour $\mathrm{t}=1$ jour). Ces deux composés ont les mêmes propriétés herbicides et pourront réagir indifféremment au cours d'un traitement phytosanitaire. L'utilisation intensive de ces produits pour usage agricole peut conduire à une contamination de l'environnement.

Le bromoxynil heptanoate est un produit très peu soluble dans l'eau $\left(s<1 \mathrm{mg}^{-1}\right)$ et le risque de le retrouver dans ce milieu est faible. Le bromoxynil a quant à lui une solubjlité qui est de plus de 100 fois supérieure à celle du bromoxynil heptanoate $\left(s=130 \mathrm{mg} \mathrm{l}^{-1}\right)$. La réaction d'hydrolyse du bromoxynil heptanoate peut donc favoriser le transfert du micropolluant organique dans l'eau et conduire à une pollution plus importante des eaux naturelles que le produit initial.

L'évolution de ces composés en milieu naturel par adsorption ou biodégradation n'a pas été étudiée, mais la faible absorption dans le visible de ces produits indique que leur élimination par photolyse directe sera faible. Le risque de retrouver ces composés dans l'eau à des teneurs supérieures à celles fixées par la directive européenne est donc envisageable.

L'étude réalisée au cours de ce travail sur l'élimination de ces deux composés par différents traitements chimiques utilisés sur des chaines de traitement d'eau potable tel que l'ozone et le chlore ou encore par des traitements photochimiques $\left(\mathrm{H}_{2} \mathrm{O}_{2} / \mathrm{UV}\right.$; $\left.\mathrm{UV}_{254}\right)$ montre que ces produits n'ont pas de rémanence dans l'eau après les différentes phases de traitement. Une disparition totale du produit initial a été observée aussi bien pour le bromoxynil que le bromoxynil heptanoate.

La comparaison des différents traitements montre que l'ozonation est probablement le traitement le plus efficace parmi tous ceux étudiés. Quelques minutes de traitement à des doses d'ozone raisonnables sont nécessaires pour éliminer le bromoxynil du milieu aqueux. Le traitement au chlore permet également une élimination de ce produit, la cinétique d'oxydation est quant à elle plus lente et nécessitera des temps de contact plus importants. La photolyse UV conduit a une bonne élimination de ces deux pesticides. Le bromoxynil et le bromoxynil heptanoate ayant un rendement quantique relativement élevé (de l'ordre de $10^{-2}$ ), l'ajout de peroxyde d'hydrogène dans le milieu n'améliore pas l'efficacité du traitement par irradiation.

La présence de sous produits d'oxydation au cours de ces différents traitements a pu être mis en évidence par l'analyse HPLC. Parmi les traitements étudiés, seuls les produits d'oxydation par $\mathrm{H}_{2} \mathrm{O}_{2} / \mathrm{UV}$ et UV ont été identifiés. Cependant, nous pouvons signaler d'après les temps de rétention observés en analyse HPLC, que certains sous-produits d'ozonation sont identiques à ceux analysés au cours des traitements par irradiation. Etant donné la grande réactivité de l'ozone vis-à-vis du produit initial et des produits de réaction, l'isolation des produits intermédiaires a été difficile et l'analyse GC-MS n'a pas permis d'identifier les produits de réaction. Mais d'après l'évolution des spectres UV des solutions ozonées, les produits de fin d'ozonation sont probablement des produits d'ouverture du cycle aromatique. Etant donné la grande réactivité de l'ozone avec cette famille de composés, les traitements 
radicalaires $\mathrm{O}_{3} / \mathrm{UV}$ et $\mathrm{O}_{3} / \mathrm{H}_{2} \mathrm{O}_{2}$ ne sont pas nécessaires pour éliminer le bromoxynil et le bromoxynil heptanoate.

L'analyse des résultats obtenus lors de l'identification des produits provenant de l'irradiation UV, nous permet de proposer un schéma réactionnel de photodégradation du bromoxynil (fig. 8). Dans ce schéma, nous avons fait apparaître le produit E, ce produit n'a pas été détecté lors de l'identification par couplage GC-MS dans notre étude, mais a été mis en évidence par KOCHANY et al. (1990 (b)).
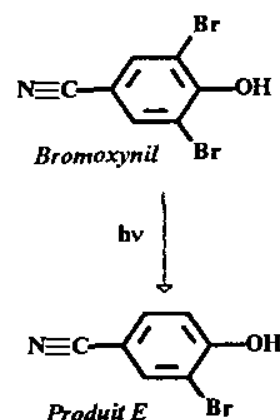

bv<smiles>N#Cc1ccc(O)cc1</smiles>

Produit $D$

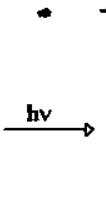<smiles>N#Cc1cc(O)c(O)c(Br)c1</smiles>

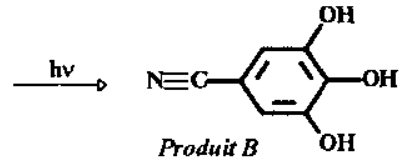

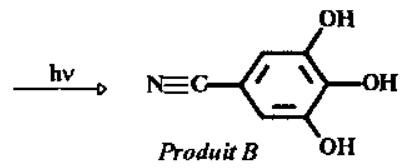

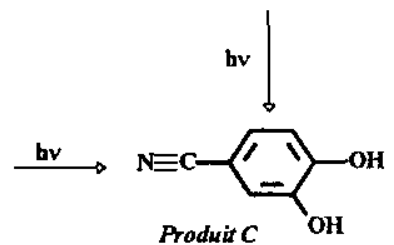

Produit $C$

Figure 8 Schéma réactionnel de photodégradation du bromoxynil heptanoate.

Photodegradation pathway of bromoxynil heptanoate by UV irradiation.

Au cours de cette étude nous avons identifié deux types de composés, des produits d'hydroxylation (produits $\mathrm{A}$ et $\mathrm{B}$ ) et des produits d'hydrogénation (produits $C$ et D). Dans les deux cas, l'attaque se fait sur les atomes de carbone bromé avec une substitution de l'atome de brome soit par un atome d'hydrogène soit par un groupement $\mathrm{OH}$.

La photolyse en milieu aqueux des produits organohalogénés conduit généralement à des dérivés où l'halogène est remplacé par un groupement $\mathrm{OH}$, mais la substitution par un atome d'hydrogène a également été observée par d'autres auteurs pour les acides chlorobenzoïques et l'amiben (CROSBY, 1966 ; PLIMMER and HUMMER, 1968).

Les mécanismes réactionnels en photolyse des composés aromatiques halogénés sont très influencés par la nature et l'orientation du substituant sur le noyau aromatique (PLIMMER, 1971). Pour de nombreux composés, les réactions de photolyse sont analogues à des réactions d'hydrolyse et conduisent à des intermédiaires hydroxylés : 


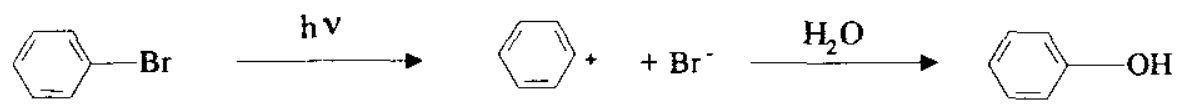

Dans le cas de composés aromatiques halogénés substitués par des groupements attracteurs d'électrons comme le bromoxynil (groupe $-\mathrm{CN}$ ), la formation des produits hydrogénés en milieu aqueux, peut s'expliquer par la coupure homolytique de la liaison $\mathrm{C}-\mathrm{X}$ au cours de la photolyse :

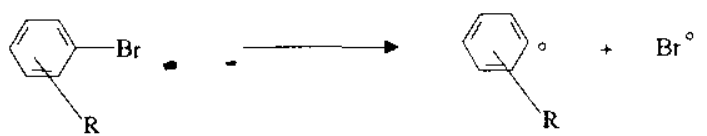

Les radicaux formés réagissent ensuite avec une molécule d'eau selon le mécanisme :<smiles>CCc1ccccc1</smiles><smiles>[R]c1ccccc1O[18F]</smiles><smiles>[R]c1ccccc1[18F]</smiles>
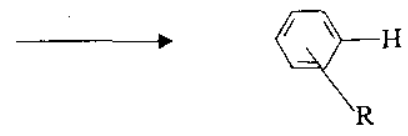

La réaction de réduction des composés aromatiques halogénés est surtout observés dans des solvants donneurs d'hydrogène comme le cyclohexane, les alcools primaire et secondaire ainsi que l'éther.

\section{CONCLUSION}

L'étude de l'oxydation du bromoxynil et du bromoxynil heptanoate en milieu aqueux par différents traitements chimiques et photochimiques a montré une bonne dégradation de ces deux pesticides aussi bien par irradiation UV que par oxydation chimique au chlore ou à l'ozone. L'analyse des sous-produits issus de l'irradiation UV a montré la formation de nombreux photoproduits dont quatre ont pu être identifiés. Ces produits n'ont pas pu être éliminés après deux heures d'irradiation.

Parmi les traitements étudiés l'ozone semble être le plus efficace aussi bien sur l'élimination des produits initiaux que sur l'élimination des produits intermédiaires. Etant donné la grande réactivité de ces deux pesticides visà-vis du chlore et de l'ozone, l'oxydation radicalaire ne semble pas être nécessaire pour l'élimination de ces composés de l'eau. 


\section{RÉFÉRENCES BIBLIOGRAPHIQUES}

BADER H., HOIGNE J. (1981). * Determina tion of ozone in water by the indigo method n. Wat. Res., 15, 449-456.

BRAUN A.M, MAURETTE M.T., OLIVEROS E. (1986). Technologie photochimique, Presses polytechniques romandes, 1 re éd., Ch II, 43-95.

BROWN D.F., MCDONOUGH L.M., McCOOL D.K., PAPENDICK R.J. (1984). "High performance liquid chromatographic determination of bromoxynil octanoate and metribuzin in runoff water from wheat fields ». J. Agric. Food Chem., 32, 195200.

CROSBY D.G. (1966). Photochemistry of herbicides, 154th Amer. Chem. Soc. Nat. Meeting, New York.

EISENBERG G. (1943). « Colorimetric deter mination of hydrogen peroxide". Ind. Eng. Chem., 15, 327-328.

KOCHANY J., CHOULDHPY G.G., WEBSTER G.R.B. (1990 (a)). « Soil organic matter chemistry II. Effect of soil fulvic acids on the environmental photodecomposition of bro. moxynil $(-3,5$ bromo -4 hydroxybenzonitrini- trile) herbicides in water ". Sci. tot. Env., 92, 181-198.

KOCHANY J., CHOUDHRY G.G., WEBSTER G.R.B (1990 (b)). "Photochemistry of halogenated derivation". Part IX « Environmental aquatic phototransformation of bromoxynil $(-3,5$ dibromo -4 hydroxybenzonitrile) *. Pest. Sci., 28, 69-81.

NICOLE I., DE LAAT J., DORE M., DUGUET J.P., BONNEL C. (1990). «Utilisation du rayonnement ultraviolet dans le traitement des eaux : mesure du flux photonique par actinométrie chimique au peroxyde d'hydrogène ". Wat. Res., 2, 157-168.

PLIMMER J.R. (1971). "The photochemistry of halogenated herbicides". Res. Rev., 33, 47-74.

PLIMMER J.R., HUMMER B.E. (1968). \& Photodegradation of 2,3,6,- trichlorobenzoic acid". Weed Sci. Soc. Amer. Abst., 20, New Orteans, La.

STANDARDISATION COMMITTEE (1987). " lodometric method for the determination of ozone in a process gas ". IOA, standardisation Committee Europe, 001/87(F). 